\title{
Phase-Resolved Detection of Ultrabroadband THz Pulses inside a Scanning Tunneling Microscope Junction
}

\author{
Melanie Müller,* Natalia Martín Sabanés, Tobias Kampfrath, and Martin Wolf
}

Cite This: ACS Photonics 2020, 7, 2046-2055

Read Online

ABSTRACT: Coupling phase-stable single-cycle terahertz ( $\mathrm{THz})$ pulses to scanning tunneling microscope (STM) junctions enables spatiotemporal imaging with femtosecond temporal and Ångstrom spatial resolution. The time resolution achieved in such $\mathrm{THz}$-gated STM is ultimately limited by the subcycle temporal variation of the tip-enhanced $\mathrm{THz}$ field acting as an ultrafast voltage pulse, and hence by the ability to feed high-frequency, broadband $\mathrm{THz}$ pulses into the junction. Here, we report on the coupling of ultrabroadband $(1-30 \mathrm{THz})$ single-cycle $\mathrm{THz}$ pulses from a spintronic

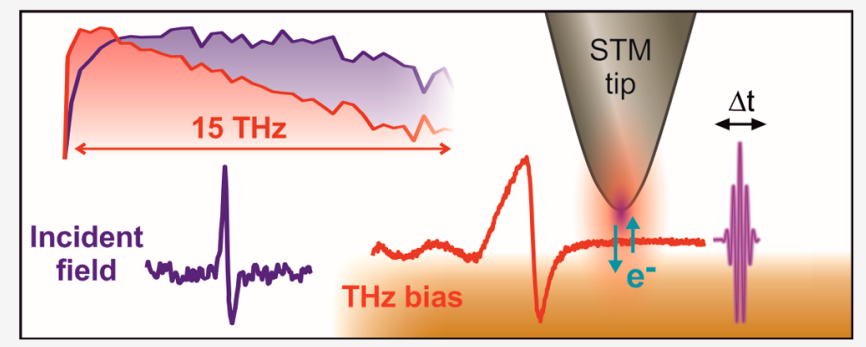
$\mathrm{THz}$ emitter (STE) into a metallic STM junction. We demonstrate broadband phase-resolved detection of the THz voltage transient directly in the STM junction via THz-field-induced modulation of ultrafast photocurrents. Comparison to the unperturbed far-field $\mathrm{THz}$ waveform reveals the antenna response of the STM tip. Despite tip-induced low-pass filtering, frequencies up to $15 \mathrm{THz}$ can be detected in the tip-enhanced near-field, resulting in $\mathrm{THz}$ transients with a half-cycle period of $115 \mathrm{fs}$. We further demonstrate simple polarity control of the THz bias via the STE magnetization and show that up to $2 \mathrm{~V} \mathrm{THz}$ bias at $1 \mathrm{MHz}$ repetition rate can be achieved in the current setup. Finally, we find a nearly constant $\mathrm{THz}$ voltage and waveform over a wide range of tip-sample distances, which by comparison to numerical simulations confirms the quasi-static nature of the $\mathrm{THz}$ pulses. Our results demonstrate the suitability of spintronic $\mathrm{THz}$ emitters for ultrafast THz-STM with unprecedented bandwidth of the THz bias and provide insight into the femtosecond response of defined nanoscale junctions.

KEYWORDS: scanning tunneling microscopy, THz voltage sampling, spintronic THz emitter, broadband THz pulses, tip antenna response, ultrafast photocurrents

$\mathrm{T}$ Hz-gated scanning tunneling microscopy (THz-STM) combines Ångstrom spatial with femtosecond temporal resolution, which has been impressively demonstrated on single molecules ${ }^{1}$ and semiconductor surfaces. ${ }^{2}$ Following the original idea of junction-mixing STM, ${ }^{3,4}$ the concept of THzSTM is based on the rectifying nature of an STM junction exhibiting nonlinear $I-V$ characteristics, leading to a net DC current upon modulation of the junction bias with ultrafast voltage pulses. Adapting this concept, THz-STM utilizes "wireless" free-space coupling of ultrafast voltage pulses to the STM by illumination with coherent broadband $\mathrm{THz}$ radiation. ${ }^{1,2,5-8}$ The STM tip hereby acts as a broadband antenna strongly enhancing the quasi-static $\mathrm{THz}$ electric field, allowing for the application of large subpicosecond bias pulses at moderate incident $\mathrm{THz}$ field strength. In contrast to ultrafast $\mathrm{THz}$ scanning near-field optical microscopy (THz-SNOM), where the scattered $\mathrm{THz}$ near-field yields information about the local transient dielectric response of the sample, ${ }^{9} \mathrm{THz}-$ STM demands that the antenna-enhanced $\mathrm{THz}$ field exhibits single-cycle character in order to ensure unambiguous control of the tunneling current within less than one optical cycle of the $\mathrm{THz}$ bias. Therefore, optimizing THz-STM operation requires precise knowledge of the $\mathrm{THz}$ voltage transient across the junction and hence broadband characterization of the tip antenna response directly in the STM environment.

The coupling of $\mathrm{THz}$ radiation to scanning probe tips has been widely studied in the context of apertureless $\mathrm{THz}$ SNOM. ${ }^{10-17}$ It is known that metallic tips act as long wire antennas, ${ }^{13,14}$ which low-pass filter the broadband incident $\mathrm{THz}$ radiation ${ }^{11}$ and exhibit highly directionally emitting and receiving properties. ${ }^{14}$ The STM tip-enhanced THz waveform will, thus, differ considerably from the incident $\mathrm{THz}$ waveform, depending on the specific STM geometry, the mesoscopic shape of the tip wire, as well as the incident $\mathrm{THz}$ spectrum. Yet, detailed experimental characterization of the STM tip antenna properties and its effect on the $\mathrm{THz}$ waveform inside the STM environment remain scarce. ${ }^{7,8}$ In particular, understanding the antenna response of the STM tip over a broad

Received: March 9, 2020

Published: July 8, 2020 

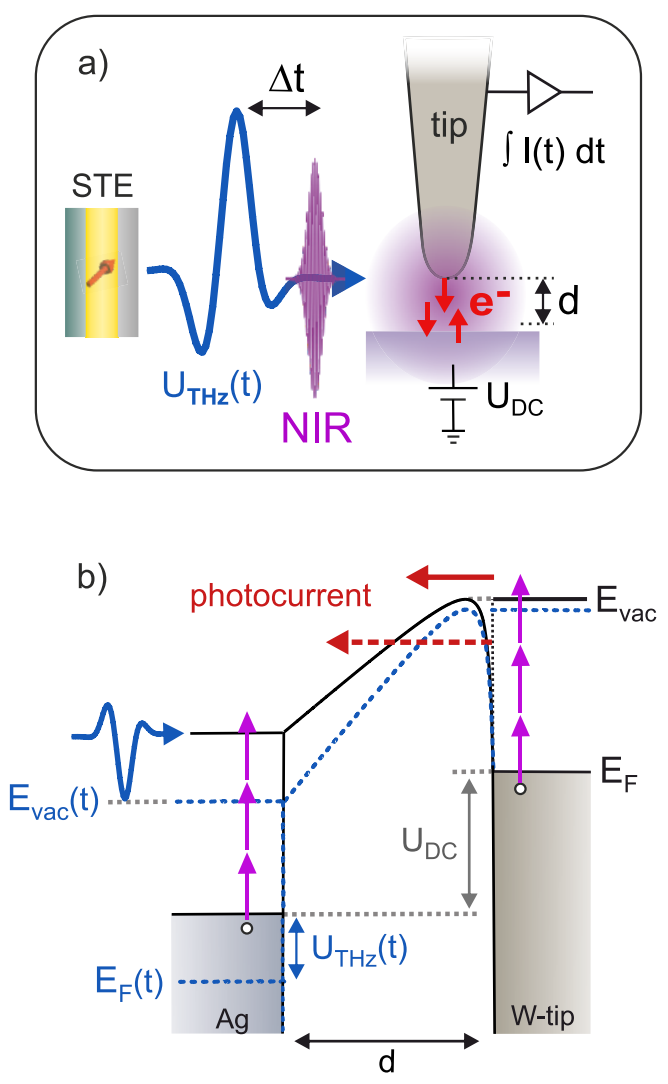

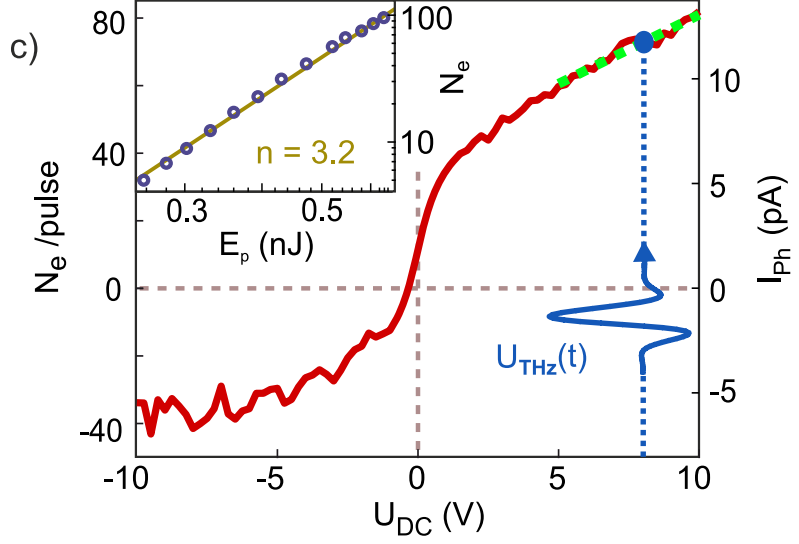

d)

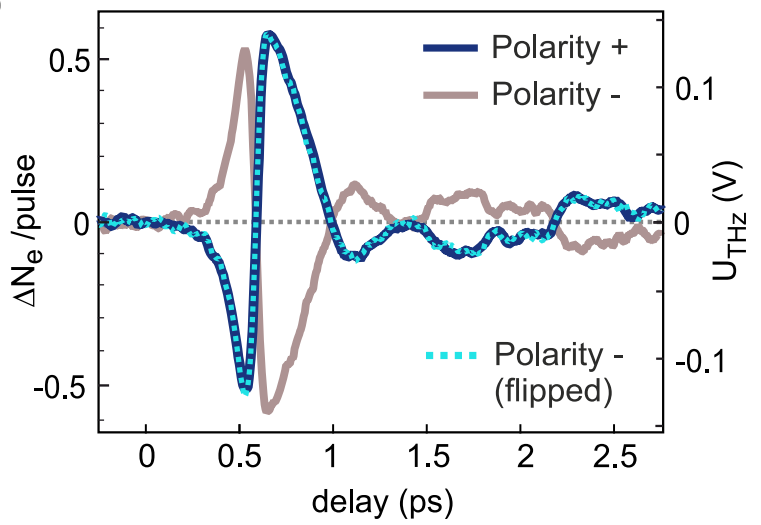

Figure 1. (a) Experimental scheme and (b) junction diagram for sampling of the THz voltage transient applied to the STM. The THz electric field emitted from the spintronic emitter (STE) acts as an instantaneous bias that modulates the photocurrent generated by 8 fs NIR laser pulses. Depending on the barrier height and distance $d$, the photocurrent originates from above-barrier photoemission and photoassisted tunneling through the barrier. (c) Laser-induced photocurrent-voltage characteristics and (d) tip-enhanced $\mathrm{THz}$ waveforms sampled at $8 \mathrm{~V}$ DC bias $(d=1$ $\mu \mathrm{m}, E_{\mathrm{p}, \mathrm{STE}}=0.6 \mu \mathrm{J}$ ) for positive and negative THz polarity (STE magnetization $\mathrm{M}+/ \mathrm{M}-$ ). The THz voltage (right $y$-axis) is retrieved from the $\mathrm{THz}$-induced photocurrent change (left $y$-axis) via the linear $I-V$ slope (green dashed line in c). The power law scaling of the photocurrent (inset in $(\mathrm{c}), U_{\mathrm{DC}}=8 \mathrm{~V}$ ) reveals the operation in the multiphoton photoemission regime.

frequency range exceeding $10 \mathrm{THz}$ will be crucial to extend the concept of THz-STM toward higher frequencies up to the multi-THz, ${ }^{9}$ mid-infrared, ${ }^{18-20}$ and even optical regime, ${ }^{21}$ potentially increasing the time resolution achievable in lightwave driven STM. To reach this goal, high-frequency singlecycle voltage transients have to be coupled into the STM with sufficient amplitude at $\mathrm{MHz}$ repetition rates, and reliable detection methods applicable to common STM setups are required for their characterization.

Recently, ultrabroadband single-cycle $\mathrm{THz}$ pulses were successfully generated from a metallic spintronic $\mathrm{THz}$ emitter (STE) with spectra covering the frequency range up to $30 \mathrm{THz}$ without gap. $^{22,23}$ The spectral bandwidth of the STE output is determined by the duration of the incident pump pulse and the resulting carrier dynamics in the STE. ${ }^{24}$ Due to its extremely large bandwidth, its fast single-cycle transients, and convenient $\mathrm{THz}$ polarity switching and polarization control via the STE magnetization, the STE is very well suited for THz-STM operation at high $\mathrm{THz}$ frequencies. In addition, it exhibits several additional advantages such as flexibility regarding pump photon energy, pulse duration, and excitation geometry, its easy handling as well as low cost. ${ }^{25}$ Although the conversion efficiency of the STE is considerably lower compared to standard $\mathrm{THz}$ sources such as $\mathrm{LiNbO}_{3}{ }^{26,27}$ or photoconductive antennas, ${ }^{28,29}$ its high beam quality in combination with high $\mathrm{THz}$ bandwidth allows for tight focusing, facilitating peak field strength of $300 \mathrm{kV} / \mathrm{cm}$ at few mJ pump pulse energies. ${ }^{23}$ Hence, high electric field strengths are achieved at comparably low $\mathrm{THz}$ power, making the STE an attractive $\mathrm{THz}$ source, in particular, for field-driven applications requiring high repetition rates such as THz-STM.

Here, we report on the generation and local detection of ultrabroadband single-cycle $\mathrm{THz}$ voltage pulses inside a metallic STM junction. We demonstrate that $\mathrm{THz}$ voltage transients with frequency components up to $15 \mathrm{THz}$ and amplitudes up to $2 \mathrm{~V}$ can be achieved at moderate pump pulse energies of a few microjoules at $1 \mathrm{MHz}$ repetition rate. To characterize the bandwidth, phase, and voltage amplitude of the tip-enhanced $\mathrm{THz}$ field, we sample its waveform directly in the time domain by $\mathrm{THz}$-induced modulation of ultrafast photocurrents excited with near-infrared (NIR) femtosecond laser pulses in the tip-sample junction, ${ }^{8,30}$ as sketched in Figure 1a,b. By comparison to the free-space $\mathrm{THz}$ waveform measured via electro-optic sampling (EOS), we can experimentally determine the receiving antenna response of the STM tip. Special care has to be taken to obtain the unperturbed tip-enhanced $\mathrm{THz}$ waveform without distortion by photoelectron dynamics, which is particularly crucial at the broad bandwidth employed here. In contrast to common nearfield detection techniques, this sampling method does not rely on scattering methods requiring demodulation techniques and a vibrating tip, which is not available in common STM setups. 
Also, it provides direct access to the $\mathrm{THz}$ voltage as the relevant quantity for STM, rather than the electric field. Finally, we analyze the distance dependence from the few nanometer to the $\mu \mathrm{m}$ range, revealing a nearly constant $\mathrm{THz}$ voltage in line with the quasi-static nature of electromagnetic radiation when applied to subwavelength dimensions. Our results demonstrate the suitability of spintronic $\mathrm{THz}$ emitters as a broadband source for the application of ultrafast voltage transients in STM, and highlight the importance of the tip antenna response that exhibits significant low-pass filtering and reduction of the $\mathrm{THz}$ bandwidth available for $\mathrm{THz}-\mathrm{STM}$ operation.

\section{EXPERIMENTAL DETAILS}

The STE is excited with broadband $800 \mathrm{~nm}$ NIR pulses of $8 \mathrm{fs}$ duration at $1 \mathrm{MHz}$ repetition rate and under normal incidence, leading to $\mathrm{THz}$ pulse emission collinear with the NIR laser beam. The STE magnetization is controlled by a permanent magnet. The STE position along the focused NIR pump beam can be set by a translation stage for variation of the NIR spot size and fluence incident on the STE. The $\mathrm{THz}$ pulses are focused onto the STM tip at $68^{\circ}$ incident angle with respect to the tip axis via an off-axis parabolic mirror $(35 \mathrm{~mm}$ focal length) integrated on the STM platform inside the ultrahigh vacuum (UHV) chamber. A second parabolic mirror is used to focus 8 fs NIR laser pulses with a pulse energy of $E_{\mathrm{p}, \mathrm{STM}}=0.6$ $\mathrm{nJ}$ into the STM (NIR spot size $\sim 6 \mu \mathrm{m}$ ) for photoexcitation of the junction at a variable time delay $\Delta t$ compared to the arrival time of the $\mathrm{THz}$ pulses. Both $\mathrm{THz}$ and NIR pulses are polarized along the tip axis. All experiments are performed at room temperature and under UHV (pressure $1 \mathrm{e}-10 \mathrm{mbar}$ ) conditions.

\section{THZ-INDUCED PHOTOCURRENT MODULATION}

Figure 1c shows the photocurrent-voltage characteristics of the photoexcited junction at a tip-sample distance of $d=1 \mu \mathrm{m}$ in the absence of the $\mathrm{THz}$ pulse. As the NIR focus is larger than the tip-sample distance, photoelectrons are excited both from the tip and the sample, and the photocurrent $I_{\mathrm{ph}}$ reverses sign at small negative DC bias. At large positive (negative) DC bias, as sketched in Figure 1b, the photocurrent is dominated by pure photoelectron emission from the tip (sample), whereas in the low bias regime it is composed of both tip and sample photoelectrons depending on their respective energy distributions. At large distances, photoemission is expected to occur either in the multiphoton regime $e^{31-33}$ or optical field-driven regime, ${ }^{34-36}$ depending on the applied NIR field strength and classified by the Keldysh parameter. ${ }^{37}$ With our experimental parameters, we obtain a Keldysh parameter in the range of $\gamma \sim$ 10-20. Hence, we clearly operate in the multiphoton photoemission regime, as confirmed by the scaling of the power dependence of the photocurrent shown in the inset in Figure 1c. We note that transient thermionic emission, that is, the emission of thermalized hot electrons, can also contribute to the photocurrent, though we expect this to be insignificant at our conditions. ${ }^{32,38,39}$ At high DC bias and, in particular, at short distances, photoassisted tunneling through the narrowed barrier $^{40}$ may also contribute to or even dominate the photocurrent.

Applying a THz pulse to the STM junction in addition to the static DC bias leads to a change in the laser-induced photocurrent due to the $\mathrm{THz}$ field. ${ }^{8,30}$ As the time scale of photoemission is given by the temporal envelope of the $8 \mathrm{fs}$ NIR laser pulse and thus occurs nearly instantaneously on the time scale of the $\mathrm{THz}$ pulse, the photoemission process is sensitive only to the instantaneous $\mathrm{THz}$ field at a given NIR-to$\mathrm{THz}$ pulse time delay $\Delta t$. One may, thus, make the quasi-static approximation, which assumes that the $\mathrm{THz}$-induced change in photocurrent will be determined by the local slope of the static $I_{\mathrm{ph}}-V$ characteristics, as sketched in Figure 1c. As will be demonstrated below, our measurements corroborate this assumption provided that noninstantaneous effects are eliminated. In the case of a linear $I_{\mathrm{ph}}-V$ curve with local slope $m_{I-V}$, as obtained at high positive bias in Figure $1 \mathrm{c}$, the instantaneous $\mathrm{THz}$ voltage $U_{\mathrm{THz}}(\Delta t)$ can be obtained from the THz-induced change in photocurrent $\Delta I_{\mathrm{THz}}$ simply by $U_{\mathrm{THz}}(\Delta t)=\Delta I_{\mathrm{THz}}(\Delta t) / m_{I-V}$. Varying the time delay $\Delta t$, thus, directly yields the calibrated $\mathrm{THz}$ voltage transient, as plotted in Figure $1 \mathrm{~d}$, measured at $8 \mathrm{~V} \mathrm{DC}$ bias and $1 \mu \mathrm{m}$ gap distance. Reversing the sign of the STE magnetization flips the $\mathrm{THz}$ polarity, ${ }^{22}$ and we find that the tip-enhanced $\mathrm{THz}$ waveform also reverses sign while maintaining its overall shape. Besides demonstrating this strikingly simple $\mathrm{THz}$ bias control in the STM provided by the STE magnetization, this finding confirms the interpretation of the employed sampling technique.

At this point, it is important to emphasize that noninstantaneous effects such as photoelectron propagation in the junction and long lifetimes of hot electrons inside the photoexcited tip and sample surface could alter the measured waveform and, thus, have to be ruled out. The lifetime of hot electrons in metals ${ }^{41}$ is in the range of a few fs to several tens of fs, depending on the sampled energy window and temperature. At large gap distances and in the absence of strong transient thermionic emission, lifetime effects can be neglected because the photoelectrons are emitted predominantly above the barrier and tunneling can occur only at the very barrier top where extremely short lifetimes of a few fs are expected. Furthermore, $\mathrm{THz}$ streaking of photoelectrons back into the tip/sample $\mathrm{e}^{30,42}$ could also result in a modified waveform, but can be precluded by operating at high $\mathrm{DC}$ bias and low $\mathrm{THz}$ field strength (smaller than the DC field). The latter is only strictly given in the single electron regime, where the electron trajectories are solely determined by the combined DC and $\mathrm{THz}$ field. At larger photocurrents with many hundreds to thousands of electrons per pulse, space charge dynamics has to be considered and can significantly alter the measured $\mathrm{THz}$ waveform by acting as an effective low-pass filter. Likewise, the excitation of long-lived hot electrons in the confined tip volume might lead to increased cooling times and thus to broadening of the measured $\mathrm{THz}$ waveform at high excitation densities. Hence, care has to be taken to operate in a lowexcitation limit at small photocurrents. We find that peak currents of 10 to a few 100 electrons per pulse are usually sufficiently low to neglect broadening effects (see Supporting Information for more detailed information). It should be noted, however, that the critical photocurrent depends on the sharpness of the tip and the net accelerating forces acting on the photoelectrons, that is, on the DC field as well as on the space charge density in the junction, given by the effective photoemission area and the tip-sample distance. To ensure instantaneous $\mathrm{THz}$ near-field sampling within the quasi-static approximation, we, thus, verify that the sampled $\mathrm{THz}$ waveform is insensitive to the $\mathrm{THz}$ polarity, incident $\mathrm{THz}$ field strength, DC bias, and tip-sample distance (details are 

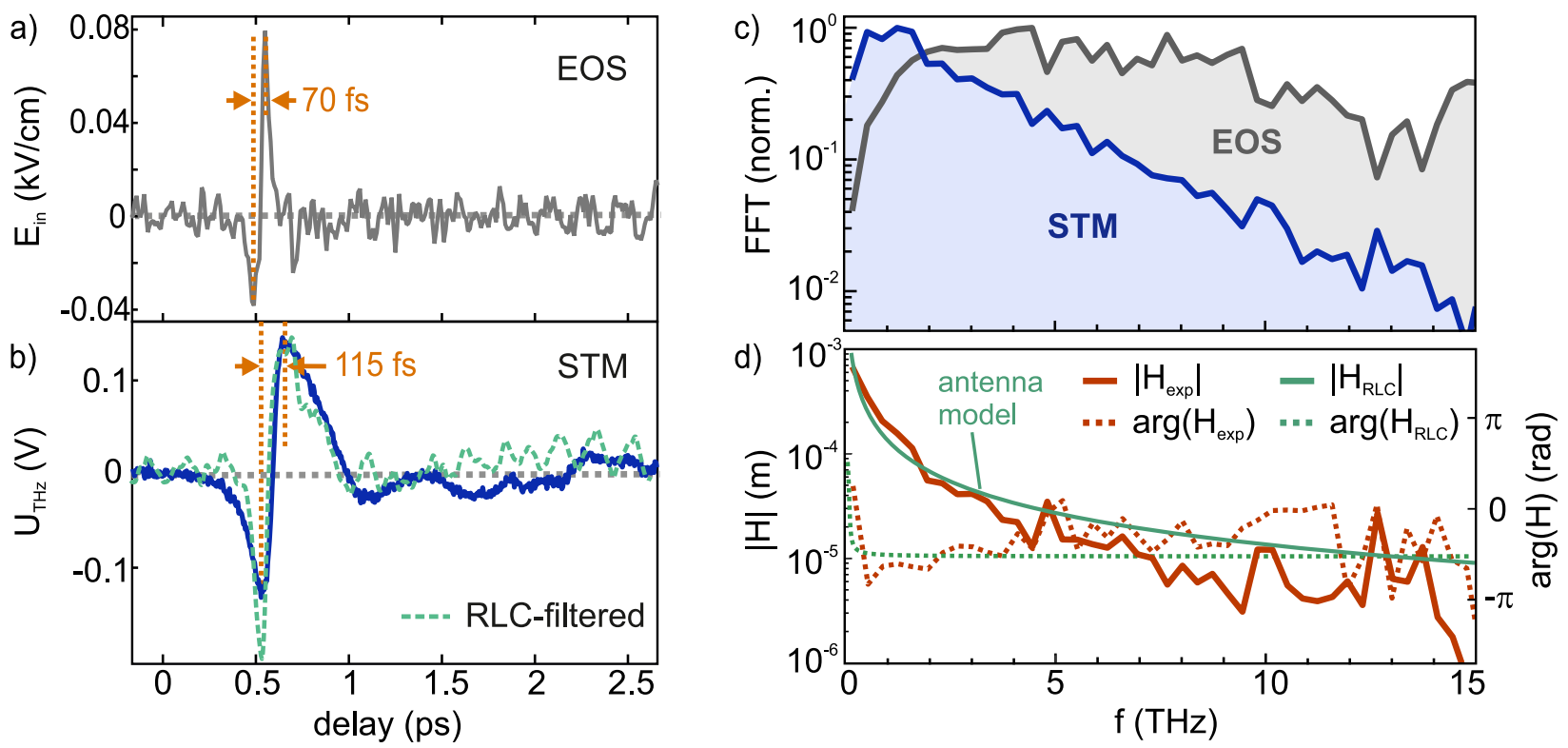

Figure 2. Comparison of (a) incident THz electric field and (b) tip-enhanced THz voltage transient in the STM junction $\left(U_{\mathrm{DC}}=8 \mathrm{~V}, d=1 \mu \mathrm{m}\right.$, $\left.E_{\mathrm{p}, \mathrm{STE}}=0.6 \mu \mathrm{J}\right)$. (c) THz amplitude spectra of the waveforms of $(\mathrm{a})$ and $(\mathrm{b})$, revealing strong low-pass filtering from the tip, which is characterized by (d) the measured receiving antenna transfer function $H(\omega)$. The green line in (d) shows the response of the antenna model with $R=300 \Omega, L=$ $0.32 \mathrm{nH}$, and $\mathrm{C}=35 \mathrm{fF}$, yielding a resonance frequency of $0.05 \mathrm{THz}$ and the $R L C$-filtered voltage transient plotted in $\mathrm{b}$ (dashed curve).

discussed in the Supporting Information and in Figure 5 below).

\section{TIP-ANTENNA RESPONSE FUNCTION}

Having established a reliable method to sample the $\mathrm{THz}$ waveform inside the STM junction, we are now able to characterize the tip antenna response by comparison of the unperturbed incident $\mathrm{THz}$ field and the received $\mathrm{THz}$ voltage transient. Figure 2a shows the $\mathrm{THz}$ electric field $E_{\text {in }}$ obtained by electro-optic sampling using a $300 \mu \mathrm{m}$ thick $\mathrm{ZnTe}(110)$ detection crystal in an identical reference beam path and after deconvolution with the detector response. ${ }^{43}$ Noise-like highfrequency features arise from the deconvolution process because the electro-optic sampling transfer function of our crystal has a relatively low amplitude at frequencies above 5 THz. Therefore, the electro-optic signal at these frequencies has lower signal-to-noise ratio. The corresponding $\mathrm{THz}$ voltage transient in the STM is shown in Figure 2b. The low-pass filter effect of the tip antenna becomes immediately obvious in the time domain data, where a half-cycle peak-to-peak separation of $115 \mathrm{fs}$ is attained in the tip-enhanced waveform, noting the maximum speed at which the $\mathrm{THz}$ bias can be reversed in the STM. In the time domain, the THz near-field in the STM junction $E_{\mathrm{STM}}(t)$ is given by convolution of the incident $\mathrm{THz}$ field $E_{\text {in }}(t)$ with the tip impulse response. More convenient access to the tip antenna response is obtained by Fourier transformation of the time domain data into the frequency domain, where the tip-enhanced $\mathrm{THz}$ field is simply the product of the incident $\mathrm{THz}$ field and the complex tip transfer function $H(\omega)$. Equivalent to a receiving antenna's output voltage and following wideband antenna theory, ${ }^{44}$ we can also use the experimentally accessible frequency-dependent $\mathrm{THz}$ voltage $U_{\mathrm{THz}}(\omega)$ instead of the tip-enhanced $\mathrm{THz}$ field and relate it to the incident $\mathrm{THz}$ electric field $E_{\text {in }}(\omega)$ by

$$
U_{\mathrm{THz}}(\omega)=H(\omega) E_{\text {in }}(\omega)
$$

Here, $H(\omega)$ is the receiving antenna transfer function whose dimension is meters. It depends on the angle $\theta$ of incidence of the $\mathrm{THz}$ beam, ${ }^{14}$ the active antenna dimensions given by the thickness and illuminated length of the STM wire, ${ }^{11}$ and geometric details of the tip. ${ }^{7}$ The Fourier spectra of the unperturbed and tip-enhanced waveforms are plotted in Figure $2 c$, revealing a pronounced bandwidth reduction and simultaneous shift of the tip-enhanced $\mathrm{THz}$ spectrum to lower frequencies. The tip transfer function $H(\omega)$ is then simply obtained by dividing the two complex Fourier spectra, and its amplitude and phase are plotted Figure $2 \mathrm{~d}$. The amplitude $|H|$ reflects the coupling efficiency of the tip antenna at a specific $\mathrm{THz}$ frequency, and its phase describes pulse distortions induced by the tip.

We model the transfer function by a simple $R L C$ circuit with resistance $R$, capacitance $C$, and inductance $L$ connected in series $^{11,30}$ (see Supporting Information for more details). The circuit parameters are adjusted to best reproduce the $\mathrm{THz}$ voltage transient from the incident waveform. We obtain reasonably good agreement for $R=300 \Omega, L=0.32 \mathrm{nH}$, and $C$ $=35 \mathrm{fF}$, yielding a resonance frequency of $f_{0}=1 /(2 \pi \sqrt{L C})=$ $0.05 \mathrm{THz}$. The $R L C$-filtered waveform and the $R L C$ transfer function are also plotted in Figure $2 \mathrm{~b}$,d. We note that the resonance is not visible here, as it lies at very low frequencies outside our $\mathrm{THz}$ spectrum. From the RLC model it becomes clear that the STM tip exhibits mostly inductive behavior in the frequency range of the STE, causing the observed low-pass filter effect. The flat spectral phase implies that no significant distortion (chirp) of the $\mathrm{THz}$ pulse is introduced by the tip due to the absence of group delay dispersion in the frequency range of the STE. Deviations of the measured and $R L C$-filtered waveforms in Figure $2 \mathrm{~b}$ are also clearly visible, indicating the limitations of the $R L C$ model to describe our results. In fact, the active antenna length, that is, the inductance and capacitance "seen" by the $\mathrm{THz}$ field, will depend on the $\mathrm{THz}$ spot size, which varies with frequency, which is not accounted for in the model. Moreover, scattering and retardation, as 

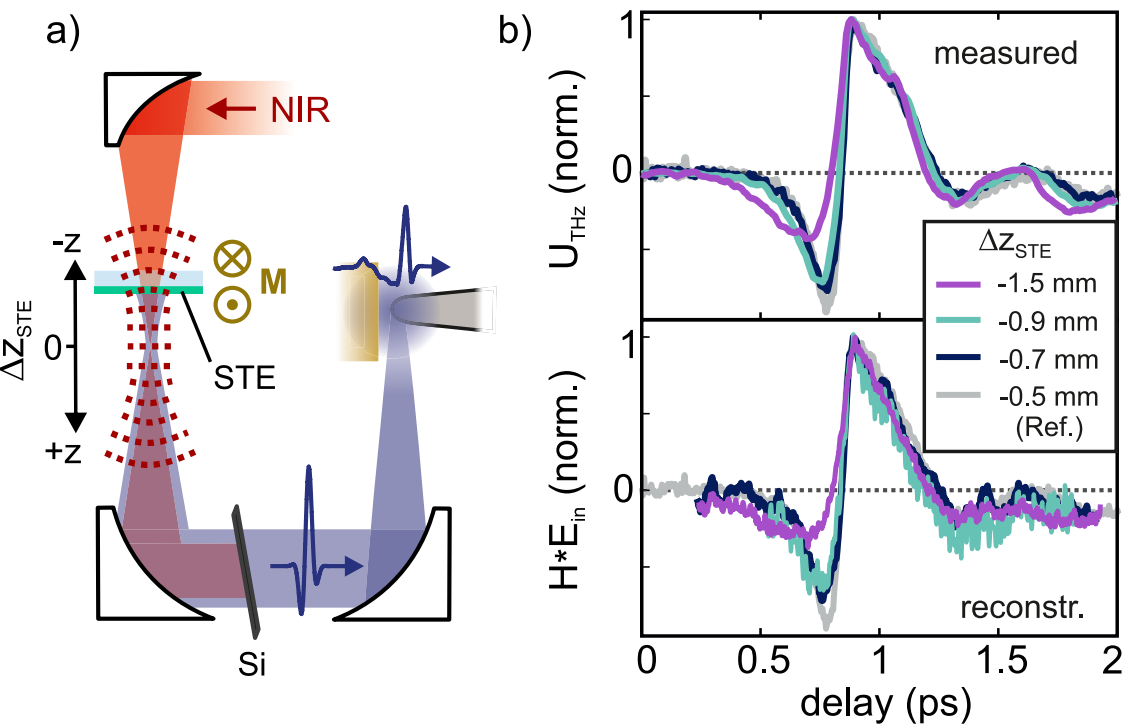

Figure 3. (a) Curved-wavefront excitation geometry with varying positions $\Delta z_{\mathrm{STE}}$ of the STE through the convergent NIR pump beam. (b) THz waveforms for various values $\Delta z_{\text {STE }}$ close to the NIR focus. The waveforms measured in the STM (top panel) are reproduced by the waveforms obtained from inverse Fourier transformation (bottom panel) of the product of the measured tip transfer function $\mathrm{H}(\omega)$ (Figure 2d) and the respective incident $\mathrm{THz}$ spectra (shown in the Supporting Information). The light gray curve shows the reference waveform from Figure 2 used to obtain $H(\omega)$. All other settings are the same as in Figure 2.
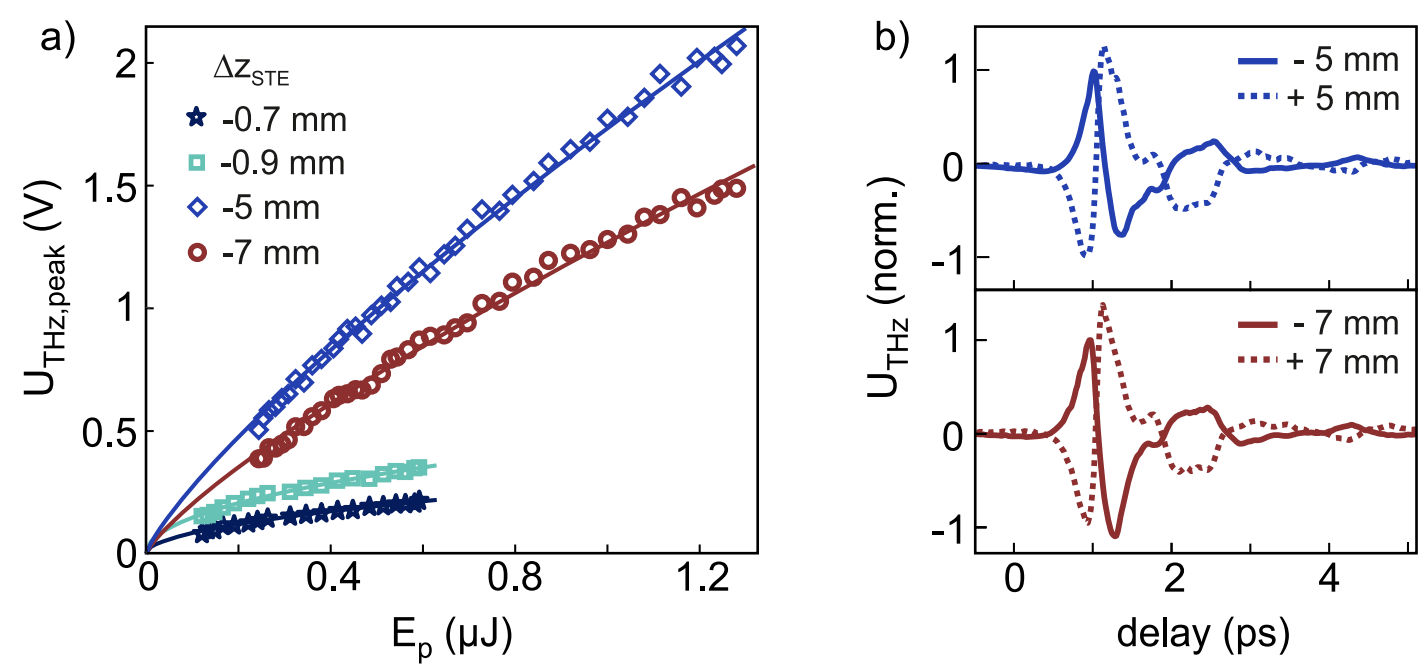

Figure 4. (a) Peak THz voltage vs STE pump pulse energy for different positions of the STE $\left(\Delta z_{\mathrm{STE}}\right)$ in front of the NIR focus. The THz voltage scales sublinearly with the pump pulse energy due to thermal saturation depending on the NIR spot size and hence STE position. Lines are sublinear fits to the data. At very small spot sizes close to the NIR focus $\left(\left|\Delta z_{\mathrm{STE}}\right|<1 \mathrm{~mm}\right)$ ablation occurs and prevents excitation at high pulse energies in the current setup. (b) THz voltage transients in the STM for two positions $\Delta z_{\mathrm{STE}}= \pm 5 \mathrm{~mm}$ and $\Delta z_{\mathrm{STE}}= \pm 7 \mathrm{~mm}$ of the $\mathrm{STE}$ far away from the NIR spot size. The dashed lines show the corresponding waveforms for the STE positioned at the same distance in the divergent NIR beam. $\left(U_{\mathrm{DC}}=8 \mathrm{~V}, d=1 \mu \mathrm{m}\right)$.

introduced by geometrical details such as the tip shaft, cannot be predicted by the RLC model. Strategies to enhance the high $\mathrm{THz}$ frequencies in the STM junction, thus, should include decreasing the inductance of the STM wire and modification of the tip transfer function via resonant tip shaping. ${ }^{7}$ We emphasize that due to the steep increase of the $\mathrm{THz}$ field enhancement at low frequencies, efficient reduction of the $\mathrm{THz}$ near-field duration requires active suppression of the low $\mathrm{THz}$ frequencies, which would otherwise dominate the response. This could be achieved by spectral matching of the incident $\mathrm{THz}$ spectrum reciprocal to the STM tip response. Finally, we note that additional low-pass filtering is caused by the frequency-dependent orientation of the antenna lobes, ${ }^{14}$ leading to less efficient coupling of the high $\mathrm{THz}$ frequencies at large incident angles with respect to the tip axis.

To demonstrate the usefulness of the transfer function, we apply a phase shift to the incident $\mathrm{THz}$ field by moving the STE inside the convergent beam of the NIR pump laser, as sketched in Figure 3a). In such a curved-wavefront excitation scheme the local radius of curvature of the NIR beam is imprinted on the generated $\mathrm{THz}$ field. ${ }^{28}$ Upon propagation to the far-field, the emitted $\mathrm{THz}$ pulse, thus, acquires a frequencydependent phase shift that depends on the STE position. In particular, an intermediate $\mathrm{THz}$ focus is expected when placing the STE in the convergent part of the NIR beam. ${ }^{28}$ As seen in the top panel in Figure $3 b$, we observe a transformation from a 
rather symmetric to a more asymmetric pulse shape of the tipenhanced $\mathrm{THz}$ waveform when moving the STE further away from the NIR focus. We then apply the measured tip transfer function to the phase-shifted incident $\mathrm{THz}$ fields and compare the reconstructed antenna-enhanced waveforms to the corresponding $\mathrm{THz}$ waveforms measured in the STM. Specifically, we multiply the complex Fourier spectra $E_{\text {in }}(\omega)$ of the deconvoluted $\mathrm{THz}$ waveforms obtained from EOS (see Figure S3 in the Supporting Information) with $H(\omega)$ to obtain the calculated Fourier spectra $U_{\text {rec }}(\omega)$ of the $\mathrm{THz}$ voltage received by the tip antenna. Inverse Fourier transformation of $U_{\text {rec }}(\omega)$ then yields the antenna-enhanced $\mathrm{THz}$ waveforms plotted in the bottom panel in Figure 3b. Comparison of the waveforms for three different STE positions reveals that the small phase shifts apparent in the measured $\mathrm{THz}$ near-field waveforms are clearly reproduced by the respective calculated waveforms, demonstrating the validity of the experimentally obtained tip transfer function. This is advantageous for routine $\mathrm{THz}$-STM operation using different $\mathrm{THz}$ waveforms, since once $H$ is known it allows to predict $\mathrm{THz}$ voltage transients in the STM from EOS reference data, given that the antenna geometry and effective length remains constant.

\section{THZ AMPLITUDE SCALING}

An important measure for the applicability of the STE for THz-STM is the achievable THz voltage in the STM junction. Figure 4a shows the peak $\mathrm{THz}$ voltage versus NIR pump pulse energy for different STE positions at $1 \mathrm{MHz}$ repetition rate. Corresponding $\mathrm{THz}$ waveforms are shown in Figures $3 \mathrm{~b}$ and 4b. We find that the $\mathrm{THz}$ voltage increases monotonically with the pump pulse energy. Peak $\mathrm{THz}$ voltages of up to $2 \mathrm{~V}$ can be achieved when placing the STE at $\Delta z_{\mathrm{STE}}=4-5 \mathrm{~mm}$ in front of the NIR focus $\left(\Delta z_{\mathrm{STE}}=0\right)$. At distances closer to the NIR focus and, thus, smaller pump spot size, the achievable $\mathrm{THz}$ field strength is reduced. We assign this behavior to thermal heating of the STE resulting in a reduced STE magnetization and hence efficiency, as well as to the decreased $\mathrm{THz}$ emission efficiency of subwavelength volumes. ${ }^{45,46}$ In addition, ablation and white light generation in the sapphire substrate prohibit the use of higher pump pulse energies at distances very close $\left(\left|\Delta z_{S T E}\right|<1 \mathrm{~mm}\right)$ to the NIR focus in the current setup. At distances far away from the NIR focus, the THz voltage in turn decreases due to the increasing NIR pump spot size (see Supporting Information) as expected from the linear fluence dependence. $^{22}$ At our laser parameters, using few-femtosecond laser pulses with microjoule energy at intermediate repetition rates of $1 \mathrm{MHz}$, the optimum excitation conditions of the STE are not yet fully explored and are subject to future work. We expect that higher $\mathrm{THz}$ voltages can be achieved by optimization of the STE excitation geometry, that is, using a weakly focused or collimated NIR pump beam. ${ }^{23}$

As seen in Figure $4 \mathrm{~b}$, the polarity of the $\mathrm{THz}$ waveform reverses sign by moving the STE from the (i) far distant convergent to the (ii) far distant divergent side of the NIR pump beam. This behavior is not unexpected because in case (i), the THz beam behind the STE exhibits an intermediate focus in which the $\mathrm{THz}$ wave undergoes a Gouy-type phase shift of $\sim 180^{\circ}$. In the region close to the NIR focus $\left(\Delta z_{\mathrm{STE}}=\right.$ $0)$, the behavior of the amplitude and shape of the emitted waveforms becomes more complex. We observe that the $\mathrm{THz}$ spectrum becomes slightly wider and the polarity of the $\mathrm{THz}$ waveform changes. The slightly enhanced detected bandwidth probably arises from the fact that the NIR focal planes at $\Delta z_{\text {STE }}$
$=0$ and at the tip position are conjugated planes that are imaged onto each other. Detailed analysis of $\mathrm{THz}$ propagation under such conditions is, however, beyond the scope of this work, and further investigations are currently in progress. Our results, thus, demonstrate that high $\mathrm{THz}$ voltages can be achieved in the STM using the STE as THz source, but point out the importance of an optimized STE excitation geometry at microjoule-level pulse energies and intermediate repetition rates as required for THz-STM.

\section{TIP-SAMPLE DISTANCE DEPENDENCE}

We finally analyze the dependence of the tip-enhanced $\mathrm{THz}$ waveform on the tip-sample distance. Figure 5a shows the dependence of the current through the STM junction versus tip-sample distance $d_{\text {rel }}$ relative to the position of a set point of the NIR-illuminated junction of $200 \mathrm{pA}$ current and $10 \mathrm{~V} \mathrm{DC}$ bias. We estimate the actual gap distance at the set point to be $4.6 \mathrm{~nm}$ (see Supporting Information for more details). Upon retraction, the current drops rapidly within the first $1 \mathrm{~nm}$. With increasing distance it stays nearly constant until at $d_{\text {rel }} \approx 600$ $\mathrm{nm}$ a pronounced photocurrent peak is observed presumably due to interference effects ${ }^{47}$ of the exciting NIR laser pulse in the junction. Whereas at far gap distances multiphoton photoemission above the broad barrier dominates, see Figure $1 \mathrm{~b}$, tunneling of photoexcited electrons through the narrowed barrier is expected to contribute significantly at close gap distances. ${ }^{48}$ At the set point distance, we most likely operate in a mixed regime of photoemission, photoassisted tunneling and DC field emission. Disentangling the different contributions requires a detailed analysis of the photocurrent nonlinearities and the potential barrier in the STM junction. ${ }^{48,49} \mathrm{THz}$ nearfield waveforms are recorded at different distances by retracting the sample by a defined step, and they are plotted in Figure $5 \mathrm{~b}$. The corresponding $I-V$ curves for calibration of the $\mathrm{THz}$ voltage are plotted in Figure 5e. Here, we note that the negative current at small positive biases arises from photoelectrons emitted with sufficient excess energy from the sample, whose contribution is enhanced at close distances as the sample moves into the center of the NIR focus. To avoid influence from drift especially at the set point distance, the feedback is temporarily switched on again between each THzNIR time delay to reference the tip position. We find that the $\mathrm{THz}$ waveform does not change considerably over a wide range of tip-sample distances. We further observe a nearly constant $\mathrm{THz}$ voltage applied to the STM junction as plotted in Figure 5c.

To better understand the scaling with tip-sample distance, we perform frequency-domain simulations of the tip-enhanced $\mathrm{THz}$ field in the junction (using the RF-module of COMSOL Multiphysics, details are described in the Supporting Information). The THz-induced potential difference $U_{\mathrm{THz}}$ applied between tip and sample can then be found by line integration of the tip-enhanced $\mathrm{THz}$ electric field $\boldsymbol{E}_{\mathrm{THz}}$ across the junction,

$$
U_{\mathrm{THz}}(\omega, d)=\int_{\text {tip }}^{\text {sample }} \boldsymbol{E}_{\mathrm{THz}}(\omega, d) \cdot \mathrm{d} \boldsymbol{r}
$$

Figure $5 \mathrm{~d}$ shows the distance scaling of the normalized THzinduced potential difference between tip and sample (solid blue curves, left ordinate) and the peak $\mathrm{THz}$ electric field (dashed blue lines, right ordinate) as obtained from simulations for three $\mathrm{THz}$ frequencies. As expected, the $\mathrm{THz}$ 
a)

c)

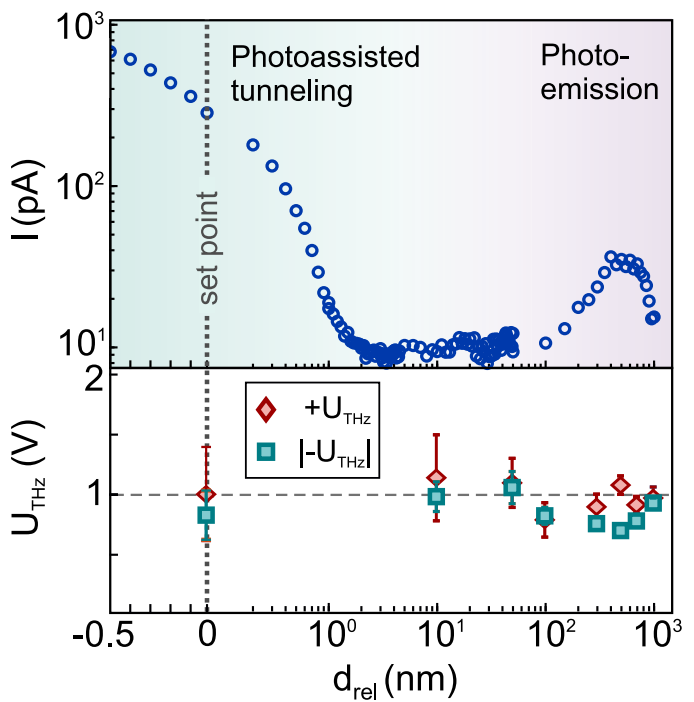

d)

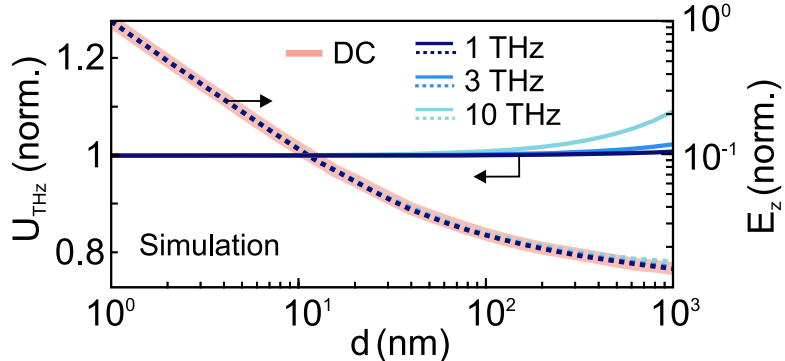

b)
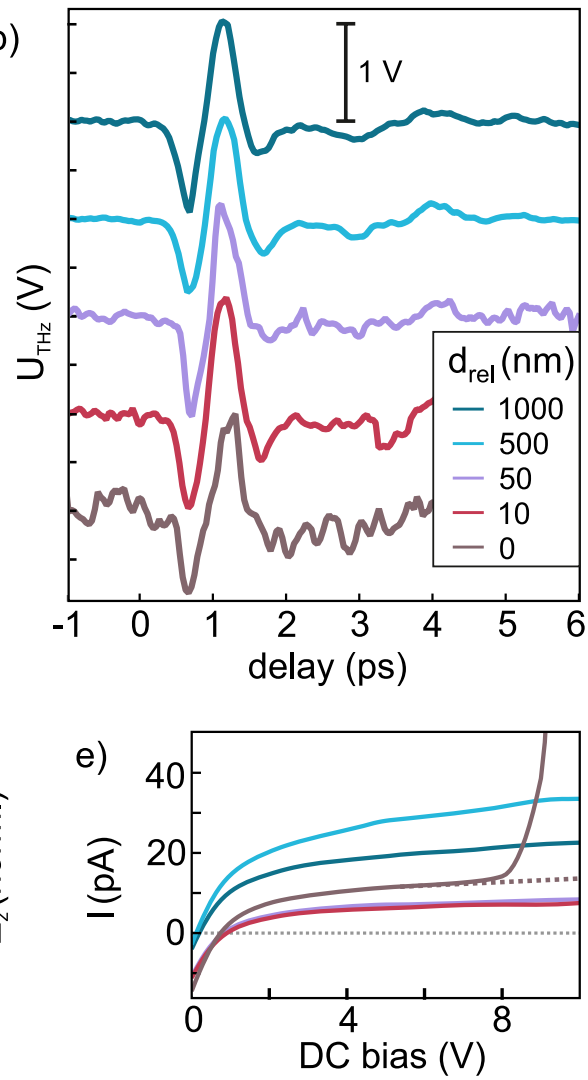

Figure 5. Dependence of the (a) photocurrent, (b) tip-enhanced THz waveform, and (c) peak THz voltage of the positive and negative half cycle on the tip-sample distance relative to a set point of $I=200 \mathrm{pA}$ and $U_{\mathrm{DC}}=10 \mathrm{~V}$. Nearly identical THz waveforms and constant THz voltages are observed. (d) Numerical simulation of the THz potential difference induced between tip and sample (blue solid lines, left $y$-axis). The THz electric field (blue dashed lines) and DC electric field (light-red solid line) in the STM junction are plotted at the right $y$-axis $\left(E_{z}\right.$ is taken $0.1 \mathrm{~nm}$ below the tip center; plotted values are normalized). (e) $I-V$ curves used for $\mathrm{THz}$ voltage calibration (waveform sampling performed at $U_{\mathrm{DC}}=8 \mathrm{~V}$ ). At $d_{\mathrm{rel}}=$ $0 \mathrm{~nm}$, the dashed line is used for calibration, which is the photocurrent $I-V$ without background from DC field emission, which is assumed to exponentially increase with bias.

electric field at the tip strongly increases at shorter distances. At the same time, we find that the $\mathrm{THz}$ voltage stays approximately constant over the investigated distance range, confirming our experimental observation and being consistent with time-domain simulations reported in previous work. ${ }^{2}$

These results imply that the $\mathrm{THz}$ electric field in the junction is quasi-static, that is, the metal surfaces of the tip and sample are equipotential surfaces for the $\mathrm{THz}$ field. Consequently, the $\mathrm{THz}$ field exhibits the same spatial distribution and distance scaling as a static field generated by a constant electric potential difference applied between tip and sample. We confirm this notion by simulation of the DC electric field, which is also plotted (light-red solid curve) in Figure $5 \mathrm{~d}$ ). We, thus, conclude that (1) retardation effects are negligible over the investigated length scale $L$, which holds if $L$ $\ll \lambda, 50$ and that (2) tip and sample behave as perfect electric conductors. Conversely, the presence of retardation effects and/or significant field penetration into the metal due to the frequency-dependent dielectric response would lead to a distance-scaling of the $\mathrm{THz}$ field that deviates from the static case, and thus a distance-dependent $\mathrm{THz}$ voltage. In fact, we find that at $1 \mathrm{THz}$, the calculated potential difference changes by less than $1 \%$ over the entire range, whereas at $10 \mathrm{THz}$, the $\mathrm{THz}$ voltage reduces by about $8 \%$ from 1000 to $1 \mathrm{~nm}$. We assign these deviations at higher $\mathrm{THz}$ frequencies and large gap distances to retardation, indicating the limitations of the quasi- static approach. Within our experimental error, our results, thus, support the view of the quasi-static nature of $\mathrm{THz}$ voltage pulses applied to nanoscale junctions, although limitations at frequencies above $10 \mathrm{THz}$ need to be further investigated.

Our observation that the $\mathrm{THz}$ waveform does not change with tip-sample distance further implies that the transfer function $H$ has a rather insignificant dependence on the gap distance, in agreement with $\mathrm{THz}$ near-field simulations reported in previous work. ${ }^{7}$ As the capacitance increases at short distances, ${ }^{15,51} \mathrm{H}$ should in general depend on the gap distance, and the antenna resonance should exhibit a red-shift at closer distances. Considering, however, that the relative increase of the capacitance is small and that the tip response is dominated by its large inductance, only marginal red-shifts of the resonance frequency are expected at the low frequency end of our $\mathrm{THz}$ spectrum, which will not have drastic effects on the $\mathrm{THz}$ near-field. The $\mathrm{THz}$ frequency response of a metallic STM junction is, thus, predominantly governed by the macroscopic wire dimensions and the $\mathrm{THz}$ focus size, defining the active antenna length.

Finally, we note that at very close gap distances the $\mathrm{THz}$ waveform might undergo additional low-pass filtering due to the increased lifetime of photoexcited electrons tunneling at lower energies through the junction. This effect does not seem to be significant at the conditions used here. Given that the undistorted waveform is precisely known, such carrier-induced 
waveform distortions will, in turn, allow one to study fewfemtosecond photocarrier dynamics with far subcycle temporal resolution and with a spatial resolution given by the localization of the photoexcited current.

In summary, we demonstrated efficient coupling of ultrabroadband single-cycle $\mathrm{THz}$ pulses from a spintronic emitter $(1-30 \mathrm{THz})$ to the junction of a scanning tunneling microscope. The STE is an attractive source for THz-STM operation, not only due its high bandwidth and fast field transients, but also due to its simple and potentially fast (tens of $\mathrm{kHz}$ ) control of the $\mathrm{THz}$ polarity and polarization via the STE magnetization. We demonstrated that $\mathrm{THz}$ voltage transients with frequencies up to $15 \mathrm{THz}$ reaching peak voltages up to $2 \mathrm{~V}$ can be coupled to the STM junction. Phaseresolved detection of the $\mathrm{THz}$ voltage transient over a wide frequency range is achieved by the THz-induced modulation of laser-excited photocurrents through the STM junction. This allows for broadband characterization of the tip transfer function directly in the STM environment. Our results show that the low-pass filtering characteristics of the STM tip antenna is a crucial parameter in the design of ultrabroadband THz-STM when aiming at increased time resolution by applying even faster $\mathrm{THz}$ voltage transients. Moreover, our experimental finding that the $\mathrm{THz}$ voltage remains constant over a wide range of tip-sample distances verifies the quasistatic nature of $\mathrm{THz}$ pulses coupled to the STM. We believe that faster voltage transients can be achieved in the STM by resonant enhancement of high $\mathrm{THz}$ frequencies via tip antenna design $^{17}$ or by blue-shifting the incident $\mathrm{THz}$ spectrum, for example, via control of the frequency-dependent propagation of the $\mathrm{THz}$ beam emitted from the STE. Modified STE excitation conditions with a collimated pump beam of suitable diameter and a subsequent $\mathrm{THz}$ telescope are expected to further increase the $\mathrm{THz}$ field amplitude incident on the tip. Easier broadband characterization of the incident $\mathrm{THz}$ electric field will be employed by using thinner electro-optic detection crystals. $^{52}$ At higher (tens of $\mathrm{THz}$ ) frequencies, we expect deviations from the quasi-static behavior due to retardation and the frequency-dependent material response. In this regard, our work provides a direct route toward the experimental characterization of the phase and amplitude of multi- $\mathrm{THz}$ voltage transients applied to an STM junction with fewfemtosecond resolution.

\section{METHODS}

Experiments are performed in an ultrahigh vacuum (UHV) system (base pressure of $<5 \times 10^{-10} \mathrm{mbar}$ ) at room temperature. The STM (customized Unisoku USM-1400 with Nanonis SPM controller) is equipped with two off-axis parabolic mirrors (PM) integrated on the spring-loaded STM platform ( $1 \times$ bare $\mathrm{Au}$ and $1 \times$ protected $\mathrm{Ag}$, $1^{\prime \prime}$ diameter, 35 $\mathrm{mm}$ focal length). The beams incident angles are $68^{\circ}$ with respect to the tip axis. The $\mathrm{THz}$ beam enters the UHV chamber via a $500 \mu \mathrm{m}$ thick diamond window and is focused by the Au mirror. NIR pulses are focused via the Ag PM for photoexcitation of the STM junction. The tip position is fixed and the sample is moved for coarse motion and scanning. The two PMs are motorized and can be moved in the $x y z$-direction (Attocube $\mathrm{GmbH}$ ) for precise focus adjustment on the tip apex. The DC bias is applied to the sample and the current is collected from the grounded tip. The current amplifier (Femto DLPCA) is operated at a gain of $10^{9} \mathrm{~V} / \mathrm{A}$ at $1 \mathrm{kHz}$ bandwidth. The THz-induced current is detected by chopping the $\mathrm{THz}$ excitation beam incident on the STE at $607 \mathrm{~Hz}$ and lock-in detection. The $\operatorname{Ag}(111)$ sample was cleaned by repeated cycles of Ar+ sputtering and annealing up to $670 \mathrm{~K}$. STM tips are electrochemically etched from $300 \mu \mathrm{m}$ thick polycrystalline tungsten wire and transferred to UHV immediately after etching.

A broadband OPCPA laser system (Venteon OPCPA, Laser Quantum) delivering 8 fs VIS-NIR laser pulses (800 $\mathrm{nm}$ center wavelength) with $3 \mu \mathrm{J}$ energy at $1 \mathrm{MHz}$ repetition rate is used for $\mathrm{THz}$ generation and photoexcitation of the STM $(2 \mu \mathrm{J}$ are available for the THz-STM setup). Part of the laser output is focused by a PM (focal length $=50 \mathrm{~mm}$ ) for $\mathrm{THz}$ generation from the STE $(5.8 \mathrm{~nm}$ thick W/CoFeB/Pt trilayer on $500 \mu \mathrm{m}$ sapphire substrate $\left.{ }^{22}\right)$ at normal incidence. The emitted $\mathrm{THz}$ radiation is collected by a second PM (focal length $=50 \mathrm{~mm}$ ) and a $500 \mu \mathrm{m}$ thick silicon wafer is used to block the collinear NIR pump beam. The $\mathrm{THz}$ beam is collinearly overlapped with 8 fs NIR pulses at a variable time delay for electro-optic sampling as well as for precise beam alignment in the STM. A $300 \mu \mathrm{m}$ thick $\mathrm{ZnTe}(110)$ crystal is used for electro-optic detection of the $\mathrm{THz}$ field outside UHV in a reference beam path that is identical to the STM beam path. The EOS signal is deconvoluted with the detector response in the time domain $^{22,43}$ to obtain the original $\mathrm{THz}$ electric field (EOS signals and deconvoluted fields are shown in the Supporting Information).

Numerical simulations are performed to calculate the $\mathrm{THz}$ near-field in the tip-sample junction by solving the timeharmonic wave equation for the electric field within the RFModule of COMSOL Multiphysics 5.5. Details are provided in the Supporting Information.

\section{ASSOCIATED CONTENT}

\section{Supporting Information}

The Supporting Information is available free of charge at https://pubs.acs.org/doi/10.1021/acsphotonics.0c00386.

Discussion of space charge effects, dependence of $\mathrm{THz}$ waveforms on $\mathrm{DC}$ bias and $\mathrm{THz}$ voltage, antenna model, measurement of incident $\mathrm{THz}$ electric fields, dependence of $\mathrm{THz}$ spectra and amplitude on STE position, estimation of tip-sample distance at the set point, and numerical simulation of $\mathrm{THz}$ electromagnetic field distributions (PDF)

\section{AUTHOR INFORMATION}

\section{Corresponding Author}

Melanie Müller - Department of Physical Chemistry, Fritz Haber Institute of the Max Planck Society, 14195 Berlin, Germany; 이이.org/0000-0002-3269-2107; Email: m.mueller@fhi-berlin.mpg.de

\section{Authors}

Natalia Martín Sabanés - Department of Physical Chemistry, Fritz Haber Institute of the Max Planck Society, 14195 Berlin, Germany; Department of Physics, Freie Universität Berlin, 14195 Berlin, Germany

Tobias Kampfrath - Department of Physical Chemistry, Fritz Haber Institute of the Max Planck Society, 14195 Berlin, Germany; Department of Physics, Freie Universität Berlin, 14195 Berlin, Germany

Martin Wolf - Department of Physical Chemistry, Fritz Haber Institute of the Max Planck Society, 14195 Berlin, Germany 
Complete contact information is available at:

https://pubs.acs.org/10.1021/acsphotonics.0c00386

\section{Notes}

The authors declare no competing financial interest.

\section{ACKNOWLEDGMENTS}

The authors thank T. Kumagai, A. Paarmann, and F. Krecinic for valuable discussions. The authors further thank $T$. Kumagai, Unisoku Inc., and M.B. Raschke for support and discussions in the development of the STM instrumentation. We thank G. Jakob and M. Kläui for providing us with the spintronic $\mathrm{THz}$ emitter and $\mathrm{H}$. Kirsch for tip preparation support. N.M.-S. and T.K. are grateful for funding through the ERC H2020 CoG Project TERAMAG/Grant No. 681917. T.K. and M.W. acknowledge the German Research Foundation (DFG) for support through Projects B02 and B07 of the SFB/ TRR227 Ultrafast Spin Dynamics.

\section{REFERENCES}

(1) Cocker, T. L.; Peller, D.; Yu, P.; Repp, J.; Huber, R. Tracking the Ultrafast Motion of a Single Molecule by Femtosecond Orbital Imaging. Nature 2016, 539 (7628), 263-267.

(2) Jelic, V.; Iwaszczuk, K.; Nguyen, P. H.; Rathje, C.; Hornig, G. J.; Sharum, H. M.; Hoffman, J. R.; Freeman, M. R.; Hegmann, F. A. Ultrafast Terahertz Control of Extreme Tunnel Currents through Single Atoms on a Silicon Surface. Nat. Phys. 2017, 13 (6), 591-597.

(3) Nunes, G.; Freeman, M. R. Picosecond Resolution in Scanning Tunneling Microscopy. Science (Washington, DC, U. S.) 1993, 262 (5136), 1029-1032.

(4) Steeves, G. M.; Elezzabi, A. Y.; Teshima, R.; Said, R. A.; Freeman, M. R. Circuit Analysis of an Ultrafast Junction Mixing Scanning Tunneling Microscope. IEEE J. Quantum Electron. 1998, 34 (8), 1415-1418.

(5) Cocker, T. L.; Jelic, V.; Gupta, M.; Molesky, S. J.; Burgess, J. A. J.; Reyes, G. D. L.; Titova, L. V.; Tsui, Y. Y.; Freeman, M. R.; Hegmann, F. A. An Ultrafast Terahertz Scanning Tunnelling Microscope. Nat. Photonics 2013, 7 (8), 620-625.

(6) Yoshioka, K.; Katayama, I.; Minami, Y.; Kitajima, M.; Yoshida, S.; Shigekawa, H.; Takeda, J. Real-Space Coherent Manipulation of Electrons in a Single Tunnel Junction by Single-Cycle Terahertz Electric Fields. Nat. Photonics 2016, 10 (12), 762-765.

(7) Yoshioka, K.; Katayama, I.; Arashida, Y.; Ban, A.; Kawada, Y.; Konishi, K.; Takahashi, H.; Takeda, J. Tailoring Single-Cycle Near Field in a Tunnel Junction with Carrier-Envelope Phase-Controlled Terahertz Electric Fields. Nano Lett. 2018, 18 (8), 5198-5204.

(8) Yoshida, S.; Hirori, H.; Tachizaki, T.; Yoshioka, K.; Arashida, Y.; Wang, Z. H.; Sanari, Y.; Takeuchi, O.; Kanemitsu, Y.; Shigekawa, H. Subcycle Transient Scanning Tunneling Spectroscopy with Visualization of Enhanced Terahertz Near Field. ACS Photonics 2019, 6 (6), 1356-1364.

(9) Eisele, M.; Cocker, T. L.; Huber, M. A.; Plankl, M.; Viti, L.; Ercolani, D.; Sorba, L.; Vitiello, M. S.; Huber, R. Ultrafast MultiTerahertz Nano-Spectroscopy with Sub-Cycle Temporal Resolution. Nat. Photonics 2014, 8 (11), 841-845.

(10) Van Der Valk, N. C. J.; Planken, P. C. M. Electro-Optic Detection of Subwavelength Terahertz Spot Sizes in the near Field of a Metal Tip. Appl. Phys. Lett. 2002, 81 (9), 1558-1560.

(11) Wang, K.; Mittleman, D. M.; Van Der Valk, N. C. J.; Planken, P. C. M. Antenna Effects in Terahertz Apertureless Near-Field Optical Microscopy. Appl. Phys. Lett. 2004, 85 (14), 2715-2717.

(12) Wang, K.; Barkan, A.; Mittleman, D. M. Propagation Effects in Apertureless Near-Field Optical Antennas. Appl. Phys. Lett. 2004, 84 (2), 305-307.

(13) Adam, A. J.; van der Valk, N. C.; Planken, P. C. Measurement and Calculation of the near Field of a Terahertz Apertureless Scanning Optical Microscope. J. Opt. Soc. Am. B 2007, 24 (5), 1080.
(14) Walther, M.; Chambers, G. S.; Liu, Z.; Freeman, M. R.; Hegmann, F. A. Emission and Detection of Terahertz Pulses from a Metal-Tip Antenna. J. Opt. Soc. Am. B 2005, 22 (11), 2357.

(15) Kersting, R.; Chen, H. T.; Karpowicz, N.; Cho, G. C. Terahertz Microscopy with Submicrometre Resolution. J. Opt. A: Pure Appl. Opt. 2005, 7 (2), S184-S189.

(16) von Ribbeck, H.-G.; Brehm, M.; van der Weide, D. W.; Winnerl, S.; Drachenko, O.; Helm, M.; Keilmann, F. Spectroscopic $\mathrm{THz}$ Near-Field Microscope. Opt. Express 2008, 16 (5), 3430.

(17) Mastel, S.; Lundeberg, M. B.; Alonso-González, P.; Gao, Y.; Watanabe, K.; Taniguchi, T.; Hone, J.; Koppens, F. H. L.; Nikitin, A. Y.; Hillenbrand, R. Terahertz Nanofocusing with Cantilevered Terahertz-Resonant Antenna Tips. Nano Lett. 2017, 17 (11), 6526-6533.

(18) Hillenbrand, R.; Taubner, T.; Keilmann, F. Phonon-Enhanced Light-Matter Interaction at the Nanometre Scale. Nature 2002, 418 (6894), 159-162.

(19) Yoshioka, K.; Igarashi, I.; Yoshida, S.; Arashida, Y.; Katayama, I.; Takeda, J.; Shigekawa, H. Subcycle Mid-Infrared Coherent Transients at $4 \mathrm{MHz}$ Repetition Rate Applicable to Light-WaveDriven Scanning Tunneling Microscopy. Opt. Lett. 2019, 44 (21), 5350.

(20) Knorr, M.; Raab, J.; Tauer, M.; Merkl, P.; Peller, D.; Wittmann, E.; Riedle, E.; Lange, C.; Huber, R. Phase-Locked Multi-Terahertz Electric Fields Exceeding $13 \mathrm{MV} / \mathrm{Cm}$ at a $190 \mathrm{kHz}$ Repetition Rate. Opt. Lett. 2017, 42 (21), 4367.

(21) Garg, M.; Kern, K. Attosecond Coherent Manipulation of Electrons in Tunneling Microscopy. Science (Washington, DC, U. S.) 2020, 367 (6476), 411-415.

(22) Seifert, T.; Jaiswal, S.; Martens, U.; Hannegan, J.; Braun, L.; Maldonado, P.; Freimuth, F.; Kronenberg, A.; Henrizi, J.; Radu, I.; Beaurepaire, E.; Mokrousov, Y.; Oppeneer, P. M.; Jourdan, M.; Jakob, G.; Turchinovich, D.; Hayden, L. M.; Wolf, M.; Münzenberg, M.; Kläui, M.; Kampfrath, T. Efficient Metallic Spintronic Emitters of Ultrabroadband Terahertz Radiation. Nat. Photonics 2016, 10 (7), $483-488$.

(23) Seifert, T.; Jaiswal, S.; Sajadi, M.; Jakob, G.; Winnerl, S.; Wolf, M.; Kläui, M.; Kampfrath, T. Ultrabroadband Single-Cycle Terahertz Pulses with Peak Fields of $300 \mathrm{KV} \mathrm{Cm}^{-1}$ from a Metallic Spintronic Emitter. Appl. Phys. Lett. 2017, 110 (25), 252402.

(24) Nenno, D. M.; Scheuer, L.; Sokoluk, D.; Keller, S.; Torosyan, G.; Brodyanski, A.; Lösch, J.; Battiato, M.; Rahm, M.; Binder, R. H.; Schneider, H. C.; Beigang, R.; Papaioannou, E. T. Modification of Spintronic Terahertz Emitter Performance through Defect Engineering. Sci. Rep. 2019, 9 (1), 13348.

(25) Fülöp, J. A.; Tzortzakis, S.; Kampfrath, T. Laser-Driven StrongField Terahertz Sources. Advanced Optical Materials; Wiley-VCH Verlag, 2020; p 1900681.

(26) Stepanov, A. G.; Hebling, J.; Kuhl, J. Efficient Generation of Subpicosecond Terahertz Radiation by Phase-Matched Optical Rectification Using Ultrashort Laser Pulses with Tilted Pulse Fronts. Appl. Phys. Lett. 2003, 83 (15), 3000-3002.

(27) Hebling, J.; Stepanov, A. G.; Almási, G.; Bartal, B.; Kuhl, J. Tunable THz Pulse Generation by Optical Rectification of Ultrashort Laser Pulses with Tilted Pulse Fronts. Appl. Phys. B: Lasers Opt. 2004, 78 (5), 593-599.

(28) Beck, M.; Schäfer, H.; Klatt, G.; Demsar, J.; Winnerl, S.; Helm, M.; Dekorsy, T. Impulsive Terahertz Radiation with High Electric Fields from an Amplifier-Driven Large-Area Photoconductive Antenna. Opt. Express 2010, 18 (9), 9251.

(29) Dreyhaupt, A.; Winnerl, S.; Helm, M.; Dekorsy, T. Optimum Excitation Conditions for the Generation of High-Electric-Field Terahertz Radiation from an Oscillator-Driven Photoconductive Device. Opt. Lett. 2006, 31 (10), 1546.

(30) Wimmer, L.; Herink, G.; Solli, D. R.; Yalunin, S. V.; Echternkamp, K. E.; Ropers, C. Terahertz Control of Nanotip Photoemission. Nat. Phys. 2014, 10 (May), 432-436. 
(31) Ropers, C.; Solli, D. R.; Schulz, C. P.; Lienau, C.; Elsaesser, T. Localized Multiphoton Emission of Femtosecond Electron Pulses from Metal Nanotips. Phys. Rev. Lett. 2007, 98 (4), 1-4.

(32) Barwick, B.; Corder, C.; Strohaber, J.; Chandler-Smith, N.; Uiterwaal, C.; Batelaan, H. Laser-Induced Ultrafast Electron Emission from a Field Emission Tip. New J. Phys. 2007, 9 (5), 142-142.

(33) Hommelhoff, P.; Kealhofer, C.; Kasevich, M. A. Ultrafast Electron Pulses from a Tungsten Tip Triggered by Low-Power Femtosecond Laser Pulses. Phys. Rev. Lett. 2006, 97 (24), 1-4.

(34) Krüger, M.; Schenk, M.; Hommelhoff, P. Attosecond Control of Electrons Emitted from a Nanoscale Metal Tip. Nature 2011, 475 (7354), 78-81.

(35) Herink, G.; Solli, D. R.; Gulde, M.; Ropers, C. Field-Driven Photoemission from Nanostructures Quenches the Quiver Motion. Nature 2012, 483 (7388), 190-193.

(36) Park, D. J.; Piglosiewicz, B.; Schmidt, S.; Kollmann, H.; Mascheck, M.; Lienau, C. Strong Field Acceleration and Steering of Ultrafast Electron Pulses from a Sharp Metallic Nanotip. Phys. Rev. Lett. 2012, 109 (24), 244803.

(37) Keldysh, L. Ionization in the Field of a Strong Electromagnetic Wave. Sov. Phys. JETP 1965, 20, 1307.

(38) Kealhofer, C.; Foreman, S. M.; Gerlich, S.; Kasevich, M. a. Ultrafast Laser-Triggered Emission from Hafnium Carbide Tips. Phys. Rev. B: Condens. Matter Mater. Phys. 2012, 86 (3), 035405.

(39) Yanagisawa, H.; Schnepp, S.; Hafner, C.; Hengsberger, M.; Kim, D. E.; Kling, M. F.; Landsman, A.; Gallmann, L.; Osterwalder, J. Delayed Electron Emission in Strong-Field Driven Tunnelling from a Metallic Nanotip in the Multi-Electron Regime. Sci. Rep. 2016, 6, 35877.

(40) Yanagisawa, H.; Hengsberger, M.; Leuenberger, D.; Klöckner, M.; Hafner, C.; Greber, T.; Osterwalder, J. Energy Distribution Curves of Ultrafast Laser-Induced Field Emission and Their Implications for Electron Dynamics. Phys. Rev. Lett. 2011, 107 (8), $1-5$.

(41) Bauer, M.; Marienfeld, A.; Aeschlimann, M. Hot Electron Lifetimes in Metals Probed by Time-Resolved Two-Photon Photoemission. Prog. Surf. Sci. 2015, 90 (3), 319-376.

(42) Herink, G.; Wimmer, L.; Ropers, C. Field Emission at Terahertz Frequencies: AC-Tunneling and Ultrafast Carrier Dynamics. New J. Phys. 2014, 16 (12), 123005.

(43) Seifert, T. S.; Jaiswal, S.; Barker, J.; Weber, S. T.; Razdolski, I.; Cramer, J.; Gueckstock, O.; Maehrlein, S. F.; Nadvornik, L.; Watanabe, S.; Ciccarelli, C.; Melnikov, A.; Jakob, G.; Münzenberg, M.; Goennenwein, S. T. B.; Woltersdorf, G.; Rethfeld, B.; Brouwer, P. W.; Wolf, M.; Kläui, M.; Kampfrath, T. Femtosecond Formation Dynamics of the Spin Seebeck Effect Revealed by Terahertz Spectroscopy. Nat. Commun. 2018, 9 (1), 1-11.

(44) Qing, X.; Chen, Z. N.; Chia, M. Y. W. Characterization of Ultrawideband Antennas Using Transfer Functions. Radio Sci. 2006, 41 (1), na.

(45) Nandi, U.; Abdelaziz, M. S.; Jaiswal, S.; Jakob, G.; Gueckstock, O.; Rouzegar, S. M.; Seifert, T. S.; Kläui, M.; Kampfrath, T.; Preu, S. Antenna-Coupled Spintronic Terahertz Emitters Driven by a 1550 Nm Femtosecond Laser Oscillator. Appl. Phys. Lett. 2019, 115 (2), 022405.

(46) Dakovski, G. L.; Kubera, B.; Shan, J. Localized Terahertz Generation via Optical Rectification in ZnTe. J. Opt. Soc. Am. B 2005, $22(8), 1667$.

(47) Raschke, M. B.; Lienau, C. Apertureless Near-Field Optical Microscopy: Tip-Sample Coupling in Elastic Light Scattering. Appl. Phys. Lett. 2003, 83 (24), 5089-5091.

(48) Schröder, B.; Bunjes, O.; Wimmer, L.; Kaiser, K.; Traeger, G. A.; Kotzott, T.; Ropers, C.; Wenderoth, M. Controlling Photocurrent Channels in Scanning Tunneling Microscopy. New J. Phys. 2020, 22 (3), 033047.

(49) Gerstner, V.; Knoll, A.; Pfeiffer, W.; Thon, A.; Gerber, G. Femtosecond Laser Assisted Scanning Tunneling Microscopy. J. Appl. Phys. 2000, 88 (8), 4851.
(50) Maier, S. A. Plasmonics: Fundamentals and Applications; Springer, 2007.

(51) Kurokawa, S.; Sakai, A. Gap Dependence of the Tip-Sample Capacitance. J. Appl. Phys. 1998, 83 (12), 7416-7423.

(52) Knorr, M.; Steinleitner, P.; Raab, J.; Gronwald, I.; Merkl, P.; Lange, C.; Huber, R. Ultrabroadband Etalon-Free Detection of Infrared Transients by van-Der-Waals Contacted Sub-10-Mm GaSe Detectors. Opt. Express 2018, 26 (15), 19059. 